\title{
Screening for Aphid Incidence on Some Potato Germplasm in Plains of West Bengal
}

\author{
Biplab Kahar ${ }^{1,2 *}$, Amitava Konar ${ }^{3}$ \\ ${ }^{1}$ Department of Plant Protection, Visva-Bharati University, Santiniketan, India \\ ${ }^{2}$ Faculty of Panchakot Mahavidyalaya, Purulia, India \\ ${ }^{3}$ Faculty of Bidhan Chandra Krishi Viswavidyalaya, Bidhan Chandra Krishi Viswavidyalaya, Mohanpur, India \\ Email: "biplab.kahar1984@gmail.com
}

Received 30 April 2016; accepted 10 June 2016; published 13 June 2016

Copyright (C) 2016 by authors and Scientific Research Publishing Inc.

This work is licensed under the Creative Commons Attribution International License (CC BY).

http://creativecommons.org/licenses/by/4.0/

(c) (i) Open Access

\begin{abstract}
A field trial was conducted to study the impact of various potato germplasm against aphids, Myzus persicae (Sulzer) and Aphis gossypii Glover (Aphididae: Hemiptera) during rabi season from November to March in 2012-2013 and 2013-2014, respectively at Adisaptagram Block Seed Farm, Hooghly, West Bengal. The population of aphids was started on potato crop in between third week of December and first week of January irrespective of various germplasms, except K. Chipsona-2, where its infestation was initiated during second and third week of January. Then, their population was gradually increased to reach its critical level (ETL) during first and second week of January in most of the potato germplasm, except in K. Anand, K. Chipsona-1, K. Chipsona-2 and Sailaja, where it was crossed in between fourth week of January and first week of February. The peak population of aphids was observed during third and fourth week of February in most of the potato germplasm. It was observed that K. Ashoka, K. Badshah, K. Chandramukhi, K. Jawahar, K. Jyoti and K. Pukhraj were highly susceptible to the pests, while K. Anand and K. Sutlez were moderately susceptible but K. Chipsona-1 , K. Chipsona-2 and K. Sailaja were less susceptible or tolerant to the pests. Maximum tuber yield ( $t / h a)$ of potato was recorded in $K$. Badshah (36.58 - 43.92) while it was lowest in $K$. Chandramukhi $(22.08-22.12)$.
\end{abstract}

Keywords

Potato, Germplasm, Aphid, Screening, West Bengal

\section{Introduction}

Potato, Solanum tuberosum L. is generally grown in all most all states in India. More than $90 \%$ of potatoes are

*Corresponding author.

How to cite this paper: Kahar, B. and Konar, A. (2016) Screening for Aphid Incidence on Some Potato Germplasm in Plains of West Bengal. Agricultural Sciences, 7, 352-356. http://dx.doi.org/10.4236/as.2016.76036 
grown in the vast Indo-Gangetic plains of North India during short winter days from October to March. Among the various states of the country, Uttar Pradesh, West Bengal and Bihar accounted for nearly $71 \%$ area and $76 \%$ production of the country (Chadha, 2002) [1]. In West Bengal, potato is one of the most important food crops next to Rice and the State ranks second position in area and production but first in the productivity (Rai, 2003) [2]. Earlier potato cultivation was largely confined to districts of Hooghly, Burdwan and West Midnapore but with the increasing facilities of irrigation, introduction of high yielding early maturing varieties and development of suitable agronomic practices potato cultivation is gradually being increased to other district of the state (Anonymous, 2005) [3]. More than 100 arthropods including insect-pests and non-insect pests infest potato crop in various parts of the world (Simpson, 1977) [4]. But Butani and Varma (1976) [5] and Misra and Agarwal (1988) [6] gave a comprehensive list of insect and non-insect pests infesting potato in India. Among these, aphid, Myzus persicae (sulzer) and Aphis gossypii Glover (Aphididae: Hemiptera), is one of the most important sucking pests, to transmit viral diseases on potato crop (Konar et al. 2001) [7]. Severe mosaic and potato leaf roll viruses were readily transmitted by aphids, while mild mosaic was partially transmitted by aphids (Khurana, 1999) [8]. These viral diseases are one of the limiting factors for virus disease free potato seed tubers production in West Bengal. Thus, farmers are bound to purchase potato seed tubers from Punjab or Himachal Pradesh by paying higher prices. Therefore, a field study was conducted to work out the effectiveness of various potato germplasms against aphids for viral disease free potato seed tubers production in the region without polluting the environment.

\section{Materials and Methods}

\subsection{Study Establishment}

A field study on the performance of different potato germplasms against aphids was carried out during rabi season from November to March of 2012-13 and 2013-14 at Adisaptagram Block Seed Farm, Hooghly, West Bengal. The experiment was laid in a Randomized Block Design (RBD) with eleven potato germplasms viz., K. Anand (K. An), K. Ashoka (K. As), K. Badshah ( K. B), K. Chandramukhi (K. Cm), K. Chipsona-1 (K. Cs 1), K. Chipsona-2 (K. Cs 2), K. Jawahar (K. Jh), K. Jyoti (K. J), K. Pukhraj (K. P), K. Sutlez (K. Su) and K. Sailaja (K. $\mathrm{Sl}$ ), each replicating for thrice. Plot size was kept at $6 \mathrm{~m} \times 2 \mathrm{~m}$ with $60 \mathrm{~cm}$ inter-row and $20 \mathrm{~cm}$ intra-row spacing. The crop was planted by the end of November in every year. All standard agronomic practices in this region were strictly followed for raising the crop, except any insecticide application. Irrespective of the germplasms, the crop was dehaulmed at an age of 85 days i.e. during first week of March and finally ten days after haulm cutting the crop was harvested.

The population of aphids on potato was recorded at 7 days interval throughout the period of study. The count on the population of aphid was made from one upper, one middle and one lower compound leaf of 33 plants in zigzag manner at random and one leaf from $34^{\text {th }}$ plant i.e., aphid population per 100 compound leaves (Simpson, 1940) [9]. The yield of healthy and damaged potato tubers were recorded at the time of harvesting for respective planting. Thus, the population dynamic of aphid and yield losses were worked out following standard method of statistical analysis.

\subsection{Data Analysis}

All the data were analyzed by different statistical analysis (Zar, 1999).

\section{Results}

In the first year of study during 2012-13, infestation of aphid was started in the third week of December in K. As, K. B, K. Cm, K. Jh, K. J and K. P. While it was by the end of December in K. Su only and it was during first week of January in K. An and K. Cs-1 (Table 1).

But in K. Cs-2 and K. Sl, the pest was observed on the crop since second week of January. Then the population of the pest was gradually increased. The pest crossed the critical level in between first and second week of January in most of the potato germplasms. In K. An and K. Cs 1, the aphid crossed the threshold limit during end January. The aphid population reached the critical level first in K. Jh during end December and last in K. Cs-2 and K. Sl during first week of February. The pest population reached the peak during third and fourth week of February in all the germplasms and then downward trend of pest population was observed. The maximum 
Table 1. (a) Population incidence of aphids on potato germplasms during 2012-13 Adisaptagram Block Seed Farm, Hooghly, West Bengal (means of three replications); (b) Source of variation.

(a)

\begin{tabular}{|c|c|c|c|c|c|c|c|c|c|c|c|c|c|}
\hline \multirow{2}{*}{$\begin{array}{l}\text { Different } \\
\text { germplasms }\end{array}$} & \multirow{2}{*}{$\begin{array}{l}\text { Tuber } \\
\text { yield } \\
\text { (t/ha) }\end{array}$} & \multicolumn{2}{|c|}{$\begin{array}{l}\text { December, } \\
2012\end{array}$} & \multicolumn{4}{|c|}{ January, 2013} & \multicolumn{4}{|c|}{ February, 2013} & \multirow{2}{*}{$\begin{array}{c}\text { March, } \\
2013 \\
\text { I WK }\end{array}$} & \multirow{2}{*}{$\begin{array}{c}\text { Mean } \\
\text { population }\end{array}$} \\
\hline & & III WK & IV WK & I WK & II WK & III WK & IV WK & I WK & II WK & III WK & IV WK & & \\
\hline K. Anand & 34.25 & $\begin{array}{c}0.0 \\
(0.0)\end{array}$ & $\begin{array}{c}0.0 \\
(0.0)\end{array}$ & $\begin{array}{c}2.66 \\
(0.44)\end{array}$ & $\begin{array}{l}7.66 \\
(0.91)\end{array}$ & $\begin{array}{l}19.33 \\
(1.28)\end{array}$ & $\begin{array}{l}54.00 \\
(1.72)\end{array}$ & $\begin{array}{l}87.33 \\
(1.93)\end{array}$ & $\begin{array}{l}124.66 \\
(2.07)\end{array}$ & $\begin{array}{l}153.66 \\
(2.15)\end{array}$ & $\begin{array}{l}207.00 \\
(2.30)\end{array}$ & $\begin{array}{l}167.33 \\
(2.20)\end{array}$ & 63.36 \\
\hline K. Ashoka & 36.21 & $\begin{array}{c}7.33 \\
(0.87)\end{array}$ & $\begin{array}{l}18.66 \\
(1.25)\end{array}$ & $\begin{array}{l}31.33 \\
(1.59)\end{array}$ & $\begin{array}{l}53.66 \\
(1.72)\end{array}$ & $\begin{array}{l}76.66 \\
(1.85)\end{array}$ & $\begin{array}{l}115.33 \\
(2.04)\end{array}$ & $\begin{array}{l}168.33 \\
(2.21)\end{array}$ & $\begin{array}{l}207.00 \\
(2.30)\end{array}$ & $\begin{array}{l}283.33 \\
(2.43)\end{array}$ & $\begin{array}{l}342.66 \\
(2.52)\end{array}$ & $\begin{array}{l}275.33 \\
(2.42)\end{array}$ & 121.28 \\
\hline K. Badshah & 36.58 & $\begin{array}{c}3.66 \\
(0.64)\end{array}$ & $\begin{array}{l}10.33 \\
(1.00)\end{array}$ & $\begin{array}{l}32.33 \\
(1.51)\end{array}$ & $\begin{array}{l}49.66 \\
(1.67)\end{array}$ & $\begin{array}{l}82.66 \\
(1.91)\end{array}$ & $\begin{array}{l}133.66 \\
(2.10)\end{array}$ & $\begin{array}{l}179.33 \\
(2.25)\end{array}$ & $\begin{array}{l}234.00 \\
(2.36)\end{array}$ & $\begin{array}{l}303.33 \\
(2.47)\end{array}$ & $\begin{array}{l}350.66 \\
(2.53)\end{array}$ & $\begin{array}{l}281.33 \\
(2.44)\end{array}$ & 127.76 \\
\hline $\begin{array}{c}\text { K. } \\
\text { Chandramukhi }\end{array}$ & 26.46 & $\begin{array}{c}5.00 \\
(0.74)\end{array}$ & $\begin{array}{l}11.66 \\
(1.07)\end{array}$ & $\begin{array}{l}24.66 \\
(1.39)\end{array}$ & $\begin{array}{l}38.00 \\
(1.57)\end{array}$ & $\begin{array}{l}69.33 \\
(1.82)\end{array}$ & $\begin{array}{l}129.66 \\
(2.09)\end{array}$ & $\begin{array}{l}181.33 \\
(2.24)\end{array}$ & $\begin{array}{l}228.33 \\
(2.35)\end{array}$ & $\begin{array}{l}286.33 \\
(2.44)\end{array}$ & $\begin{array}{l}324.33 \\
(2.49)\end{array}$ & $\begin{array}{l}249.00 \\
(2.38)\end{array}$ & 119.05 \\
\hline $\begin{array}{c}\text { K. } \\
\text { Chipsona-I }\end{array}$ & 22.17 & $\begin{array}{c}0.0 \\
(0.0)\end{array}$ & $\begin{array}{c}0.0 \\
(0.0)\end{array}$ & $\begin{array}{c}2.66 \\
(0.46)\end{array}$ & $\begin{array}{c}6.66 \\
(0.81)\end{array}$ & $\begin{array}{l}11.33 \\
(1.05)\end{array}$ & $\begin{array}{l}27.33 \\
(1.42)\end{array}$ & $\begin{array}{l}68.66 \\
(1.82)\end{array}$ & $\begin{array}{l}97.66 \\
(1.95)\end{array}$ & $\begin{array}{l}131.66 \\
(2.09)\end{array}$ & $\begin{array}{l}169.33 \\
(2.20)\end{array}$ & $\begin{array}{l}112.33 \\
(2.02)\end{array}$ & 48.28 \\
\hline $\begin{array}{c}\text { K. } \\
\text { Chipsona-II }\end{array}$ & 26.92 & $\begin{array}{c}0.0 \\
(0.0)\end{array}$ & $\begin{array}{c}0.0 \\
(0.0)\end{array}$ & $\begin{array}{c}0.0 \\
(0.0)\end{array}$ & $\begin{array}{l}3.00 \\
(0.49)\end{array}$ & $\begin{array}{c}6.66 \\
(0.84)\end{array}$ & $\begin{array}{l}12.33 \\
(1.07)\end{array}$ & $\begin{array}{l}29.33 \\
(1.43)\end{array}$ & $\begin{array}{l}56.33 \\
(1.70)\end{array}$ & $\begin{array}{l}79.66 \\
(1.84)\end{array}$ & $\begin{array}{l}126.66 \\
(2.08)\end{array}$ & $\begin{array}{l}76.33 \\
(1.85)\end{array}$ & 30.02 \\
\hline K. Jawahar & 27.87 & $\begin{array}{c}6.00 \\
(0.79)\end{array}$ & $\begin{array}{l}21.66 \\
(1.33)\end{array}$ & $\begin{array}{l}56.66 \\
(1.74)\end{array}$ & $\begin{array}{l}87.33 \\
(1.93)\end{array}$ & $\begin{array}{l}127.33 \\
(2.09)\end{array}$ & $\begin{array}{l}169.33 \\
(2.22)\end{array}$ & $\begin{array}{l}227.00 \\
(2.35)\end{array}$ & $\begin{array}{l}276.66 \\
(2.43)\end{array}$ & $\begin{array}{l}341.33 \\
(2.52)\end{array}$ & $\begin{array}{c}393.33 \\
(2.58)\end{array}$ & $\begin{array}{l}286.33 \\
(2.44)\end{array}$ & 153.30 \\
\hline K. Jyoti & 26.50 & $\begin{array}{c}3.66 \\
(0.53)\end{array}$ & $\begin{array}{c}8.66 \\
(0.94)\end{array}$ & $\begin{array}{l}17.33 \\
(1.24)\end{array}$ & $\begin{array}{l}30.33 \\
(1.47)\end{array}$ & $\begin{array}{l}56.66 \\
(1.71)\end{array}$ & $\begin{array}{l}97.33 \\
(1.97)\end{array}$ & $\begin{array}{l}143.33 \\
(2.14)\end{array}$ & $\begin{array}{l}212.66 \\
(2.31)\end{array}$ & $\begin{array}{l}269.66 \\
(2.42)\end{array}$ & $\begin{array}{c}244.33 \\
(2.37)\end{array}$ & $\begin{array}{l}164.33 \\
(2.19)\end{array}$ & 96.02 \\
\hline K. Pukhraj & 22.12 & $\begin{array}{c}4.33 \\
(0.65)\end{array}$ & $\begin{array}{c}9.33 \\
(0.97)\end{array}$ & $\begin{array}{l}20.33 \\
(1.31)\end{array}$ & $\begin{array}{l}38.33 \\
(1.58)\end{array}$ & $\begin{array}{l}61.66 \\
(1.76)\end{array}$ & $\begin{array}{l}94.66 \\
(1.96)\end{array}$ & $\begin{array}{l}147.33 \\
(2.16)\end{array}$ & $\begin{array}{l}192.33 \\
(2.27)\end{array}$ & $\begin{array}{c}244.00 \\
(2.37)\end{array}$ & $\begin{array}{l}172.66 \\
(2.21)\end{array}$ & $\begin{array}{l}107.33 \\
(1.99)\end{array}$ & 84.02 \\
\hline K. Sutlez & 25.79 & $\begin{array}{c}0.0 \\
(0.0)\end{array}$ & $\begin{array}{c}2.66 \\
(0.44)\end{array}$ & $\begin{array}{l}10.66 \\
(1.02)\end{array}$ & $\begin{array}{l}31.33 \\
(1.49)\end{array}$ & $\begin{array}{l}48.33 \\
(1.66)\end{array}$ & $\begin{array}{l}72.33 \\
(1.85)\end{array}$ & $\begin{array}{l}114.33 \\
(2.04)\end{array}$ & $\begin{array}{l}169.00 \\
(2.22)\end{array}$ & $\begin{array}{l}234.33 \\
(2.35)\end{array}$ & $\begin{array}{l}196.66 \\
(2.27)\end{array}$ & $\begin{array}{l}147.66 \\
(2.14)\end{array}$ & 79.02 \\
\hline K. Sailaja & 33.25 & $\begin{array}{c}0.0 \\
(0.0)\end{array}$ & $\begin{array}{c}0.0 \\
(0.0)\end{array}$ & $\begin{array}{c}0.0 \\
(0.01)\end{array}$ & $\begin{array}{c}2.33 \\
(0.43)\end{array}$ & $\begin{array}{c}8.66 \\
(0.95)\end{array}$ & $\begin{array}{l}15.33 \\
(1.17)\end{array}$ & $\begin{array}{l}31.66 \\
(1.46)\end{array}$ & $\begin{array}{l}57.66 \\
(1.73)\end{array}$ & $\begin{array}{l}83.33 \\
(1.87)\end{array}$ & $\begin{array}{c}152.33 \\
(2.16)\end{array}$ & $\begin{array}{c}104.66 \\
(1.99)\end{array}$ & 35.07 \\
\hline
\end{tabular}

(b)

\begin{tabular}{cccc}
\hline & Date of observation & Variety & Date of observation $\times$ variety \\
\hline SEM $( \pm)$ & 0.03 & 0.03 & 0.11 \\
C.D. $-0.05 \%$ & 0.07 & 0.07 & 0.25 \\
\hline
\end{tabular}

*Figures in parenthesis are logarithmic transformed values.

population of aphid was found in K. Jh (393.33 per 100 compound leaves), followed by K. B (350.66), K. As (342.66), K. Cm (324.33), K. J (269.66), K. P (244.0), K. Su (234.33), K. An (207.0), K. Cs-1 (169.33), K. Sl (152.33) and K. Cs 2 (126.66), respectively. The mean population of the pest was recorded highest in K. Jh (153.30 per 100 compound leaves) and then K. B (127.76), K. As (121.28), K. Cm (107.87), K. J (96.02), K. P (84.02), K. Su (79.02), K. An (63.36), K. Cs 1 (48.28), K. Sl (35.07) and K. Cs 2 (30.02), respectively. Yield of healthy tubers of potato (t/ha) was found maximum in $\mathrm{K}$. B (36.58) while it was recorded minimum in $\mathrm{K}$. $\mathrm{P}$ (22.12).

In the second year of study during 2013-14, the initial infestation of aphid was recorded during end of December in most of the germplasms (Table 2).

The pest was observed slightly earlier in K. B, K. Jh and K. Su in third week of December, while it was found quite later in K. An and K. Cs 1 during first week of January. But in case of K. Cs 2 and K. Sl, aphid was recorded from second week of January. Aphid population crossed the critical level in between first and second week of January in most of the potato germplasms, except K. An, K. Cs 1, K. Cs 2 and K. Sl. In K. Cs 2, the threshold limit of the pest of was reached in third week of February, which was quite late in respect to others. The peak period of the pest was not only found in between third and fourth week of February, but it was also 
Table 2. (a) Population incidence of aphids on potato germplasms during 2013-14 Adisaptagram Block Seed Farm, Hooghly, West Bengal (means of three replications); (b) Source of variation.

(a)

\begin{tabular}{|c|c|c|c|c|c|c|c|c|c|c|c|c|c|}
\hline \multirow{2}{*}{$\begin{array}{c}\text { Different } \\
\text { germplasms }\end{array}$} & \multirow{2}{*}{$\begin{array}{l}\text { Tuber } \\
\text { yield } \\
\text { (t/ha) }\end{array}$} & \multicolumn{2}{|c|}{$\begin{array}{c}\text { December, } \\
2013\end{array}$} & \multicolumn{4}{|c|}{ January, 2014} & \multicolumn{4}{|c|}{ February, 2014} & \multirow{2}{*}{$\begin{array}{c}\text { March, } \\
2014 \\
\text { I WK }\end{array}$} & \multirow{2}{*}{$\begin{array}{c}\text { Mean } \\
\text { population }\end{array}$} \\
\hline & & III WK & IV WK & I WK & II WK & III WK & IV WK & I WK & II WK & III WK & IV WK & & \\
\hline K. Anand & 40.25 & $\begin{array}{c}0.0 \\
(0.0)\end{array}$ & $\begin{array}{c}0.0 \\
(0.0)\end{array}$ & $\begin{array}{c}3.66 \\
(0.53)\end{array}$ & $\begin{array}{c}8.66 \\
(0.95)\end{array}$ & $\begin{array}{l}15.66 \\
(1.18)\end{array}$ & $\begin{array}{l}37.66 \\
(1.57)\end{array}$ & $\begin{array}{l}62.00 \\
(1.79)\end{array}$ & $\begin{array}{l}91.33 \\
(1.95)\end{array}$ & $\begin{array}{l}133.33 \\
(2.12)\end{array}$ & $\begin{array}{l}167.33 \\
(2.21)\end{array}$ & $\begin{array}{l}192.66 \\
(2.28)\end{array}$ & 54.79 \\
\hline K. Ashoka & 39.83 & $\begin{array}{c}0.0 \\
(0.0)\end{array}$ & $\begin{array}{c}5.66 \\
(0.76)\end{array}$ & $\begin{array}{l}16.33 \\
(1.21)\end{array}$ & $\begin{array}{l}30.33 \\
(1.48)\end{array}$ & $\begin{array}{l}64.66 \\
(1.81)\end{array}$ & $\begin{array}{l}96.66 \\
(1.98)\end{array}$ & $\begin{array}{l}137.33 \\
(2.13)\end{array}$ & $\begin{array}{l}192.00 \\
(2.28)\end{array}$ & $\begin{array}{l}276.66 \\
(2.44)\end{array}$ & $\begin{array}{c}337.33 \\
(2.52)\end{array}$ & $\begin{array}{l}281.33 \\
(2.45)\end{array}$ & 110.64 \\
\hline K. Badshah & 43.92 & $\begin{array}{c}2.33 \\
(0.49)\end{array}$ & $\begin{array}{c}9.66 \\
(1.00)\end{array}$ & $\begin{array}{l}29.33 \\
(1.45)\end{array}$ & $\begin{array}{l}62.33 \\
(1.79)\end{array}$ & $\begin{array}{l}93.00 \\
(1.97)\end{array}$ & $\begin{array}{r}159.66 \\
(2.20)\end{array}$ & $\begin{array}{l}214.66 \\
(2.33)\end{array}$ & $\begin{array}{c}271.66 \\
(2.43)\end{array}$ & $\begin{array}{l}316.33 \\
(2.50)\end{array}$ & $\begin{array}{c}377.33 \\
(2.58)\end{array}$ & $\begin{array}{l}294.66 \\
(2.47)\end{array}$ & 140.84 \\
\hline K. Chandramukhi & 27.37 & $\begin{array}{c}0.0 \\
(0.0)\end{array}$ & $\begin{array}{c}4.33 \\
(0.68)\end{array}$ & $\begin{array}{l}13.66 \\
(1.14)\end{array}$ & $\begin{array}{l}31.66 \\
(1.50)\end{array}$ & $\begin{array}{l}72.33 \\
(1.86)\end{array}$ & $\begin{array}{r}108.33 \\
(2.02)\end{array}$ & $\begin{array}{l}153.33 \\
(2.18)\end{array}$ & $\begin{array}{c}209.33 \\
(2.32)\end{array}$ & $\begin{array}{l}287.66 \\
(2.46)\end{array}$ & $\begin{array}{c}217.33 \\
(2.33)\end{array}$ & $\begin{array}{l}162.66 \\
(2.20)\end{array}$ & 96.97 \\
\hline K. Chipsona-I & 29.67 & $\begin{array}{c}0.0 \\
(0.0)\end{array}$ & $\begin{array}{c}0.0 \\
(0.0)\end{array}$ & $\begin{array}{c}2.66 \\
(0.44)\end{array}$ & $\begin{array}{c}8.33 \\
(0.91)\end{array}$ & $\begin{array}{l}13.33 \\
(1.11)\end{array}$ & $\begin{array}{l}19.66 \\
(1.28)\end{array}$ & $\begin{array}{l}32.66 \\
(1.50)\end{array}$ & $\begin{array}{l}69.66 \\
(1.83)\end{array}$ & $\begin{array}{l}102.33 \\
(2.00)\end{array}$ & $\begin{array}{l}146.33 \\
(2.16)\end{array}$ & $\begin{array}{l}212.00 \\
(2.06)\end{array}$ & 39.69 \\
\hline K. Chipsona-II & 30.00 & $\begin{array}{c}0.0 \\
(0.0)\end{array}$ & $\begin{array}{c}0.0 \\
(0.0)\end{array}$ & $\begin{array}{c}0.0 \\
(0.0)\end{array}$ & $\begin{array}{c}3.33 \\
(0.50)\end{array}$ & $\begin{array}{c}7.33 \\
(0.87)\end{array}$ & $\begin{array}{l}12.66 \\
(1.10)\end{array}$ & $\begin{array}{l}18.66 \\
(1.26)\end{array}$ & $\begin{array}{l}27.66 \\
(1.43)\end{array}$ & $\begin{array}{l}51.33 \\
(1.67)\end{array}$ & $\begin{array}{l}78.33 \\
(1.87)\end{array}$ & $\begin{array}{l}109.33 \\
(2.02)\end{array}$ & 23.74 \\
\hline K. Jawahar & 37.54 & $\begin{array}{c}5.66 \\
(0.76)\end{array}$ & $\begin{array}{c}9.33 \\
(0.98)\end{array}$ & $\begin{array}{l}17.33 \\
(1.24)\end{array}$ & $\begin{array}{l}33.33 \\
(1.51)\end{array}$ & $\begin{array}{l}68.33 \\
(1.83)\end{array}$ & $\begin{array}{l}89.00 \\
(1.95)\end{array}$ & $\begin{array}{l}137.00 \\
(2.13)\end{array}$ & $\begin{array}{l}192.66 \\
(2.28)\end{array}$ & $\begin{array}{l}263.33 \\
(2.41)\end{array}$ & $\begin{array}{l}312.33 \\
(2.49)\end{array}$ & $\begin{array}{l}247.33 \\
(2.39)\end{array}$ & 105.82 \\
\hline K. Joti & 30.17 & $\begin{array}{c}0.0 \\
(0.0)\end{array}$ & $\begin{array}{c}3.33 \\
(0.58)\end{array}$ & $\begin{array}{l}10.66 \\
(1.03)\end{array}$ & $\begin{array}{l}23.33 \\
(1.37)\end{array}$ & $\begin{array}{l}51.33 \\
(1.71)\end{array}$ & $\begin{array}{l}83.33 \\
(1.92)\end{array}$ & $\begin{array}{l}122.33 \\
(2.08)\end{array}$ & $\begin{array}{l}187.66 \\
(2.27)\end{array}$ & $\begin{array}{l}249.66 \\
(2.39)\end{array}$ & $\begin{array}{l}193.66 \\
(2.28)\end{array}$ & $\begin{array}{l}136.66 \\
(2.13)\end{array}$ & 81.69 \\
\hline K. Pukhraj & 22.08 & $\begin{array}{c}0.0 \\
(0.0)\end{array}$ & $\begin{array}{c}6.33 \\
(0.83)\end{array}$ & $\begin{array}{l}18.66 \\
(1.27)\end{array}$ & $\begin{array}{l}24.33 \\
(1.39)\end{array}$ & $\begin{array}{l}42.33 \\
(1.62)\end{array}$ & $\begin{array}{l}68.33 \\
(1.83)\end{array}$ & $\begin{array}{l}93.33 \\
(1.97)\end{array}$ & $\begin{array}{l}127.66 \\
(2.10)\end{array}$ & $\begin{array}{l}168.66 \\
(2.21)\end{array}$ & $\begin{array}{l}217.33 \\
(2.32)\end{array}$ & $\begin{array}{l}172.66 \\
(2.27)\end{array}$ & 72.28 \\
\hline K. Sutlez & 32.17 & $\begin{array}{c}3.00 \\
(0.56)\end{array}$ & $\begin{array}{c}7.00 \\
(0.86)\end{array}$ & $\begin{array}{l}16.66 \\
(1.22)\end{array}$ & $\begin{array}{l}29.33 \\
(1.46)\end{array}$ & $\begin{array}{l}57.33 \\
(1.76)\end{array}$ & $\begin{array}{l}89.66 \\
(1.95)\end{array}$ & $\begin{array}{l}124.33 \\
(2.09)\end{array}$ & $\begin{array}{c}163.66 \\
(2.21)\end{array}$ & $\begin{array}{c}209.33 \\
(2.32)\end{array}$ & $\begin{array}{c}253.66 \\
(2.40)\end{array}$ & $\begin{array}{l}187.33 \\
(2.26)\end{array}$ & 87.79 \\
\hline K. Sailaja & 38.50 & $\begin{array}{c}0.0 \\
(0.0)\end{array}$ & $\begin{array}{c}0.0 \\
(0.0)\end{array}$ & $\begin{array}{c}0.0 \\
(0.0)\end{array}$ & $\begin{array}{c}3.66 \\
(0.64)\end{array}$ & $\begin{array}{c}7.66 \\
(0.88)\end{array}$ & $\begin{array}{l}16.33 \\
(1.19)\end{array}$ & $\begin{array}{l}43.33 \\
(1.63)\end{array}$ & $\begin{array}{c}75.66 \\
(1.88)\end{array}$ & $\begin{array}{l}109.33 \\
(2.03)\end{array}$ & $\begin{array}{c}129.33 \\
(2.10)\end{array}$ & $\begin{array}{l}163.33 \\
(2.21)\end{array}$ & 42.20 \\
\hline
\end{tabular}

(b)

\begin{tabular}{cccc}
\hline & Date of observation & Variety & Date of observation $\times$ variety \\
\hline SEM $( \pm)$ & 0.02 & 0.02 & 0.08 \\
C.D. $0.05 \%$ & 0.05 & 0.05 & 0.19 \\
\hline
\end{tabular}

*Figures in parenthesis are logarithmic transformed values.

extended up to first week of March in K. An, K. Cs 2 and K. Sl. The mean aphid population was found highest in K. B (140.84 per 100 compound leaves) and in the order were K. As (110.64), K. Jh (105.82), K. Cm (96.97), K. Su (87.79), K. J (81.69), K. P (72.28), K. An (54.79), K. Sl (42.20), K. Cs 1 (39.69) and K. Cs 2 (23.74), respectively.

\section{Discussion}

It is revealed from the present field study that the aphids were appeared on potato crop in between third week of December and first week of January irrespective of different germplasms, except K. Cs 2, where its infestation was initiated during second and third week of January. After appearance on the crop pest population was gradually increased and continued up to dehaulming of the crop. In all germplasms the economic threshold limit of the pest was observed during first and second week of January in most of the potato germplasms, when temperature ranged from $7.90^{\circ} \mathrm{C}-27.20^{\circ} \mathrm{C}$, relative humidity $(\mathrm{RH}) 48.70 \%-100.0 \%$, bright sunshine (BSS) duration 4.70 $8.40 \mathrm{~h}$ and wind speed from $0.40-1.3 \mathrm{~km} / \mathrm{h}$. But in K. An, K. Cs 1, K. Cs 2 and K. Sl, it was crossed in between fourth week of January and first week of February. The peak population of the pest was recorded during third and fourth week of February under climatic condition of $15.50^{\circ} \mathrm{C}-33.10^{\circ} \mathrm{C}$ temperature, $39.90 \%-99.30 \% \mathrm{RH}$, 
7.60 - $10.10 \mathrm{~h}$ BSS and $1.00-1.40 \mathrm{~km} / \mathrm{h}$ wind speed.

The low aphid population in K. Cs 2 was may be due to its morphological characters, since the leaves of the plant were rough, coarse narrower as well as smaller than other germplasms. The taller habit of the plant, perhaps, also made an unfavourable microclimatic condition within the crop canopy as the pest population was positively correlated with relative humidity and negatively with minimum temperature during rabi season (Sontakke et al. 1989) [10]. Konar and Paul (2004) [11] also reported that K. B, K. Cm and K. J were more susceptible to the pest as compared to K. An and K. Su. It has also been observed that the bottom leaves of most of germplasms maintained the highest aphid population while the upper leaves maintained the lowest, suggesting that the aphids have marked preference for senescing leaves under shady condition (Nderitu and Mueke, 1989) [12].

\section{Conclusion}

It may be concluded from the present field investigation that $\mathrm{K}$. As, K. B, K. Cm, K. Jh, K. J and K. P were highly susceptible; K. An \& K. Su were moderately susceptible and K. Cs 1, K. Cs 2 and K. Sl were less susceptible or tolerant to pest.

\section{Acknowledgements}

The authors are thankful to the Farm Manager of Adisaptagram Block Seed Farm, Hooghly, West Bengal for providing various facilities including a piece of land to undertaken the present field study.

\section{References}

[1] Chadha, K.L. (2002) In: Handbook of Horticulture. ICAR, New Delhi, 8-10.

[2] Rai, M. (2003) Role of Potato in Food and Nutritional Security in Developing Countries with Special Reference to India. Proceeding of the National Symposium "Potato Research towards National Food and Nutritional Security", CPRI, Shimla H.P, 19-30.

[3] Anonymous (2005) Annual Report AICRP on Potato. BCKV, Kalyani Nadia, 32-34.

[4] Simpson, G.W. (1977) Potato Insects and Their Control. In: Smith, O., Ed., Potatoes Production, Storage and Processing, AVI Publishing Co., Westport, 550-605.

[5] Butani, D.K. and Verma, S. (1976) Pest of Vegetable and Their Control-3: Potato. Pesticides, 10, 46-51.

[6] Misra, S.S. and Agrawal, H.O. (1988) Potato Pests in India and There Control. Tropical Pest Management, 34, 199209. http://dx.doi.org/10.1080/09670878809371242

[7] Konar, A., Basu, A., Mukhopadhyay, S.K. and Chettri, M. (2001) Population Builds up of Aphids on Potato in Burdwan District of West Bengal. Journal of Indian Potato Association, 28, 123-124.

[8] Khurana, S.P.M. (1999) In: Shimla, H.P., Eds., Potato Viruses and Viral Diseases, CPRI (ICAR), Technical Bulletin No-35 (Revised), 2-5.

[9] Simpson, S.W. (1940) Aphid and Their Relation to the Field Transmission of Potato Viral Diseases in North-East Maine. Maine Agricultural Experiment-Statistical Bulletin, 403.

[10] Sontakke, B.K., Singh, D.N. and Misra, B. (1889) Seasonal Abundance of Potato Pests in Relation to Environmental Factors. Environment and Ecology, 7, 391-394.

[11] Konar, A. and Paul, S. (2004) Population Builds up of Aphids on Different Potato Genotypes in Gangetic Plains of West Bengal. Abstract of the National Symposium on "Aphids in Agriculture and Forestry", University of Kalyani, Nadia, 28.

[12] Nderitu, J.H. and Mueke, J.M. (1989) Susceptibility of Eight Potato Cultivars to Myzus persicae Sulzer in the Greenhouse. Insect Science and Its Application, 10, 229-234. 